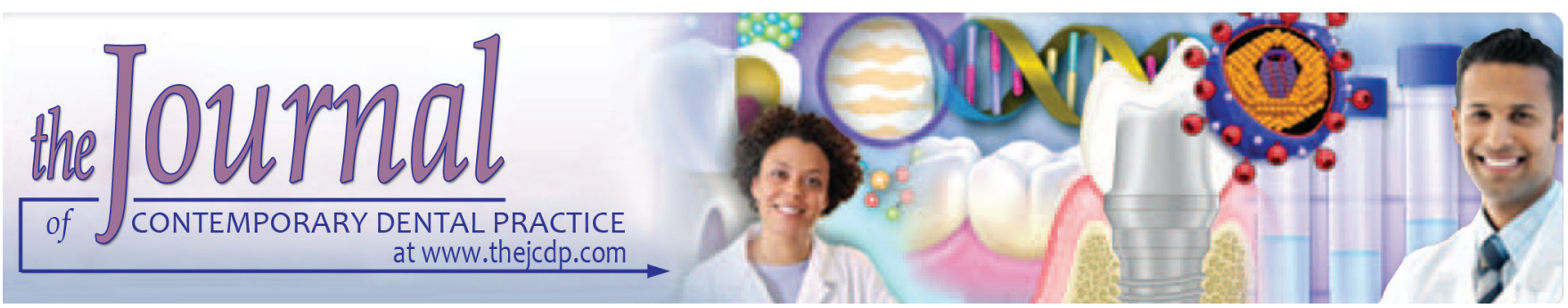

\title{
Influence of Three-dimensional Imaging on Implant Treatment Planning: Implant Diameter and Length
}

\author{
${ }^{1}$ Dorothea C Dagassan-Berndt, ${ }^{2}$ Walter Clemens, ${ }^{3}$ Nicola U Zitzmann, ${ }^{4}$ Ralf K Schulze
}

\begin{abstract}
Aim: To investigate the influence of the radiographic modalities panoramic radiography $(\mathrm{PR})$ and cone beam computed tomography $(\mathrm{CBCT})$ on selection of implant length and diameter.

Materials and methods: Potential implant sites (59 sites, 40 patients) with available PRs and CBCTs were selected from the databases of two University dental schools. Six experienced clinicians were asked to select the appropriate implant length and diameter based on PR and CBCT. Results were compared with the actual surgical procedure and intra- /interrater agreement was calculated using Cohen's kappa.
\end{abstract}

Results: On average, greater implant length was more often planned based on PR (24.2 vs $8.8 \%$ with CBCT), while wider implants were generally more frequently planned based on CBCTs (19.9 vs $29.7 \%$ with PR). Implant treatment planning based on PR and on CBCT images was equal in 50 to $67 \%$. Compared with the actual surgery, CBCT revealed a higher agreement (46\% for implant length), while for PR, $34.4 \%$ equal implant length was observed.

Conclusion: The radiographic modality used for implant treatment planning affects implant selection.

Clinical significance: The CBCT scans are advantageous for choosing the appropriate implant dimension. Caution should be exercised when selecting implant length planned from PR.

\footnotetext{
${ }^{1}$ Department of Oral Surgery, Oral Radiology and Oral Medicine University Center of Dental Medicine Basel, University of Basel Basel, Switzerland

${ }^{2}$ Department of Periodontology, Endodontology, and Cariology University Center of Dental Medicine Basel, University of Basel Basel, Switzerland

${ }^{3}$ Department of Reconstructive Dentistry, University Center of Dental Medicine Basel, University of Basel, Basel, Switzerland

${ }^{4}$ Department of Oral Surgery and Oral Radiology, University Medical Center of the Johannes Gutenberg-University Mainz Mainz, Germany

Corresponding Author: Dorothea C Dagassan-Berndt Department of Oral Surgery, Oral Radiology and Oral Medicine University Center of Dental Medicine Basel, University of Basel Basel, Switzerland, Phone: +41612672606, e-mail: dorothea. dagassan@unibas.ch
}

Keywords: Cone beam computed tomography, Dental implants, Implant treatment planning, Panoramic radiographs.

How to cite this article: Dagassan-Berndt DC, Clemens W, Zitzmann NU, Schulze RK. Influence of Three-dimensional Imaging on Implant Treatment Planning: Implant Diameter and Length. J Contemp Dent Pract 2018;19(6):704-711.

\section{Source of support: Nil}

Conflict of interest: None

\section{INTRODUCTION}

Panoramic radiography is the standard two-dimensional (2D) imaging technique applied prior to any surgical procedure in dentistry. ${ }^{1,2}$ During the last two decades three-dimensional (3D) imaging, particularly CBCT, has been established to visualize potential implant sites in more detail. While both methods are deemed to be accurate for implant treatment planning, differences exist with respect to visualization, effective dose, costs, and availability of the apparatus. ${ }^{3-7}$ Magnification rates and distortion of PR may lead to difficulties in measuring bone height and width. A safety margin of $2 \mathrm{~mm}$ has been established for dental implants to ensure integrity of adjacent anatomical structures. ${ }^{8}$ The CBCT offers a more detailed visualization, e.g., of the mandibular canal, ${ }^{9}$ and a reduced safety zone of $1.7 \mathrm{~mm}$ to this nerve structure has been recommended. ${ }^{10}$ If implants are requested in areas with a potential high risk of damaging vital structures or with deficient bone morphologies, implant guidelines indicate 3D imaging with CBCT scans for more detailed information. ${ }^{6,11}$

The benefit of diagnostic imaging for the patient has been discussed and graded in six levels, i.e., the efficacy of imaging methods. ${ }^{12}$ While levels 1 to 3 are related to technical quality, diagnostic accuracy efficacy, and "diagnostic thinking efficacy," level 4 assesses the effect on the treatment plan. Levels 5 and 6 measure the effect on patient outcomes, and, finally, the analysis of societal 
costs. Most studies about implant treatment planning remain on levels 1 or $2 .{ }^{13}$ Recent studies reported about level three and documented a difference of implant treatment planning based on 2D and 3D images. ${ }^{14-17}$ Providing more detailed bone height measurements in $\mathrm{CBCT}$, bone augmentation was more accurately predicted from CBCT compared with PR. ${ }^{16}$ The potential effect of 3D visualization on implant selection in relation to the surgical protocol has not yet been evaluated.

The aim of the current study was to examine the effect of different imaging modalities (PR vs CBCT) on the selection of implant dimensions (implant length and diameter) in terms of diagnostic thinking efficacy (level 3).

\section{MATERIALS AND METHODS}

\section{Study Sample}

Records of 40 patients ( 24 women and 16 men, mean age 49 years, 19-78 years) requesting single tooth implants in an edentulous space were selected from two clinics (Departments for Oral Surgery, Basel, Switzerland, and Mainz, Germany). The study was approved by the Ethics Research Committee of the University of Basel Switzerland (EK: 245/12). Patients' records included preoperative digital PRs and a CBCT scan from the intended implant site. The PRs and CBCT scans were available to the respective surgeon before implant placement. Records on the implant dimensions (length and diameter) used during the actual surgery existed as inclusion criteria. A total of 59 implant sites in the premolar or molar region of the upper $(n=27)$ and lower $(n=32)$ jaw were included.

\section{Image Acquisition}

The PRs were acquired with two different machines: Cranex D (Soredex, Tuusula, Finland) or Orthophos XG (Sirona Dental Systems GmbH, Bensheim, Germany). The CBCT scans were made either with the Accuitomo
80 (J Morita Corp., Kyoto, Japan) with a voxel size of $0.125 \mathrm{~mm}$ or with the 3D Exam (Kavo Dental, Biberach, Germany) with a voxel size of $0.2 \mathrm{~mm}$. All images were exported as DICOM files in their natural resolution, and subsequently imported to the Osirix-DICOM-Viewer (www.osirix-viewer.com). ${ }^{16}$

\section{Observers and Observation Process}

Six observers, experienced in dental implantology (five oral surgeons, one specialist in prosthodontics), were recruited to analyze the cases. Prior to the evaluation and planning process, the observers were calibrated with respect to the applications and features of the DICOMViewer. All 2D and 3D images were presented on a computer screen (size $1680 \times 1050$, Intel HD Graphics 3000 $384 \mathrm{MB}$ ) in full volume size and with the option to change all possible setting options (adjusting the contrast, scrolling through the volume, etc.). The screen was calibrated with a software program (Dental Q, Luxor Display Q, IBA Dosimetry, Schwarzenbruck, Germany). All images were presented in a DICOM-Viewer (Osirix, v.4.1.2.). During the entire viewing period, the study supervisor (D.C.D.) recorded the observers' results and guided them through the analyzing process. ${ }^{13}$ A total of 80 images (40 PR and 40 CBCT scans) were evaluated twice (one repetition) by each observer (Figs 1 and 2). While evaluation

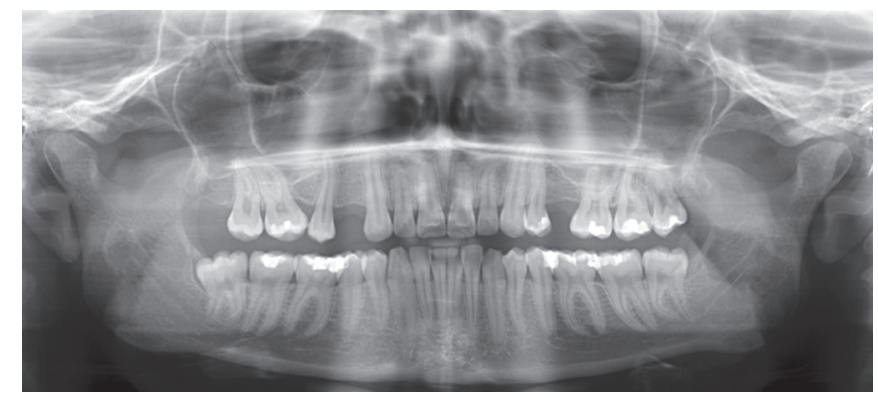

Fig. 1: Panoramic radiograph for implant planning area 14

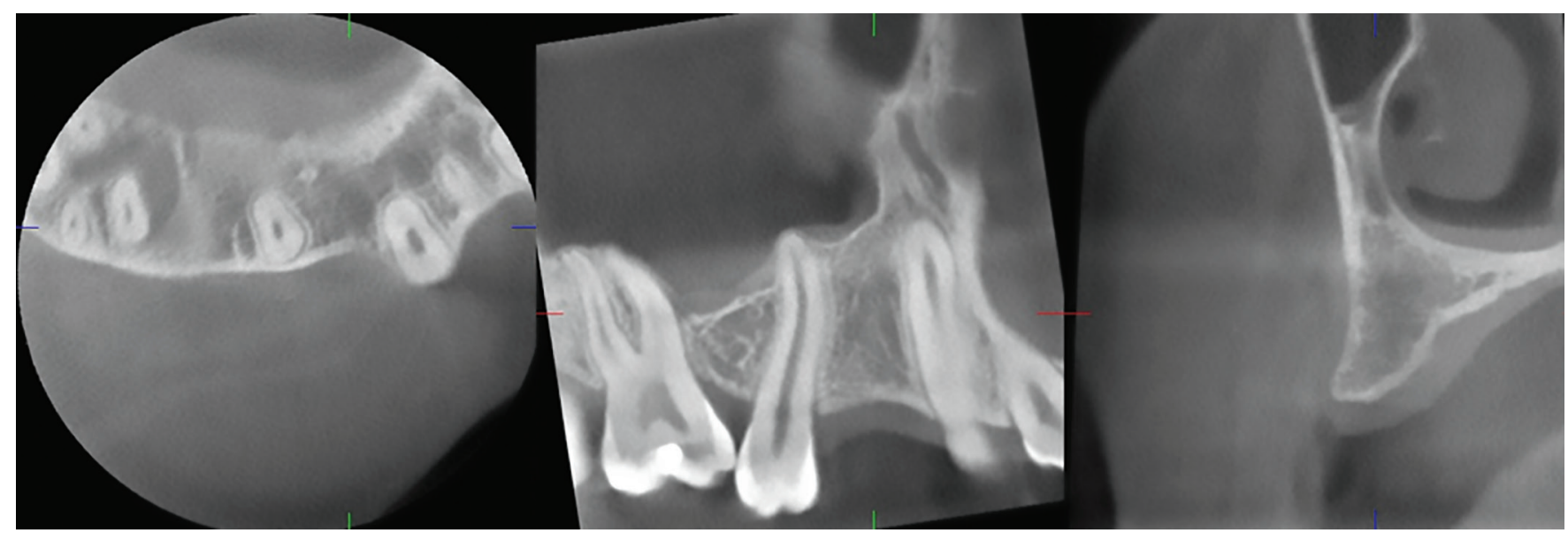

Fig. 2: Cone beam computed tomography image for implant planning area 14 
Table 1: Analysis of implant selection based on PRs and CBCT

\begin{tabular}{llllllll}
\hline & & $\begin{array}{l}\text { PR planning } \\
\text { "longer/wider" }\end{array}$ & $\begin{array}{l}\text { Diameter not } \\
\text { defined on PR }\end{array}$ & $\begin{array}{l}\text { CBCT planning } \\
\text { "longer/wider" }\end{array}$ & $\begin{array}{l}\text { Diameter not } \\
\text { defined on CBCT }\end{array}$ & Both equally & $p$-value* \\
\hline Procedure & Jaw & & & & & & \\
Implant length & Maxilla $\mathrm{n}=324$ & $19.8 \%(64)$ & 0 & $10.5 \%(34)$ & 0 & $69.8 \%(226)$ & 0.134 \\
& Mandible $\mathrm{n}=384$ & $27.9 \%(107)$ & 0 & $7.3 \%(28)$ & 0 & $64.8 \%(249)$ & 0.001 \\
& Both jaws $\mathrm{n}=708$ & $24.2 \%(171)$ & 0 & $8.8 \%(62)$ & 0 & $67.1 \%(475)$ & 0 \\
Implant & Maxilla $\mathrm{n}=324$ & $13.6 \%(24)$ & 148 & $34.1 \%(60)$ & 0 & $52.3 \%(92)$ & 0.625 \\
diameter & Mandible $\mathrm{n}=384$ & $25.1 \%(54)$ & 169 & $26.0 \%(56)$ & 0 & $48.8 \%(105)$ & 0.002 \\
& Both jaws $\mathrm{n}=708$ & $19.9 \%(78)$ & 317 & $29.7 \%(116)$ & 0 & $50.4 \%(197)$ & 0.092 \\
\hline
\end{tabular}

${ }^{*} p$-value relates to difference between methods related to invasiveness of planning

of each radiograph was conducted without time limits, the evaluation time of one session was restricted to 1.5 hours to avoid excessive observer fatigue. Four sessions were required by each observer. Sessions were separated by a minimum period of two weeks to ensure that observers were not recalling their initial decisions.

\section{Planning Implant Dimensions}

The observers were asked to evaluate the images at the given implant sites including neighboring structures, and to measure the maximum bone height (in $\mathrm{mm}$ ) to plan the implant dimensions. ${ }^{16}$ Observers were informed about the (global) magnification factor provided by the manufacturers for PR, i.e., for the Cranex D 1:1.25, and for the Orthophos XG, 1:1.19. Applying these global magnification factors, the readings of the observers were subsequently corrected.

Observers were asked to select one of the following options for implant length:

- No implant possible

- $6 \mathrm{~mm}$ implant length

- $8 \mathrm{~mm}$ implant length

- $10 \mathrm{~mm}$ implant length

- $12 \mathrm{~mm}$ implant length

In addition, implant diameter was determined from CBCT using the following options:

- No implant possible

- Implant planning not evaluable

- $3.3 \mathrm{~mm}$ implant diameter

- $4.1 \mathrm{~mm}$ implant diameter

- $4.8 \mathrm{~mm}$ implant diameter

At the stage of designing this study, it was assumed that it would not be reasonable to estimate an implant diameter solely based on PR due to the unreliable horizontal magnification in PRs. During conduct of the study, several observers (yet not all of them) still provided diameter estimations on PRs. Since these data were now available for some cases, this information was included in the analysis. This explains the reduced number of cases for this specific parameter.

\section{Data Processing and Statistical Analysis}

The number of disagreements between CBCT and PR for the outcomes (selected implant length and diameter) was reported with counts and percentages (Table 1). Any deviations between the two imaging methods or in comparison with the surgical procedure were considered as "more invasive" as soon as a longer and/or wider implant dimension was planned. Correspondingly, a treatment plan was graded as "less invasive," when smaller implant dimensions were planned, or "equal" with concurring plans or decisions. Ratings with no planning were excluded. To accommodate for potential pooled data, the most frequent values (mode) of implant length and diameter were determined for each site and imaging method. The modes of different methods (PR and $\mathrm{CBCT}$ ) were compared using McNemar-Bowker test or the Wilcoxon signed-rank test for paired data. Prior to testing, categories with few entries were merged as appropriate. Further, the outcomes (implant length and diameter) based on PR or CBCT were compared with the actually conducted surgical procedure and displayed with counts and percentages (Fig. 3 and Table 2).

In a first analysis, the implant parameter of the actual surgical procedure was compared with both imaging methods. All data were considered to calculate the modes of the imaging methods (PR and CBCT), which were compared with the conducted surgical procedure and categorized into "equal" and "nonequal." Secondly, the "invasiveness" of the methods (CBCT and PR) was

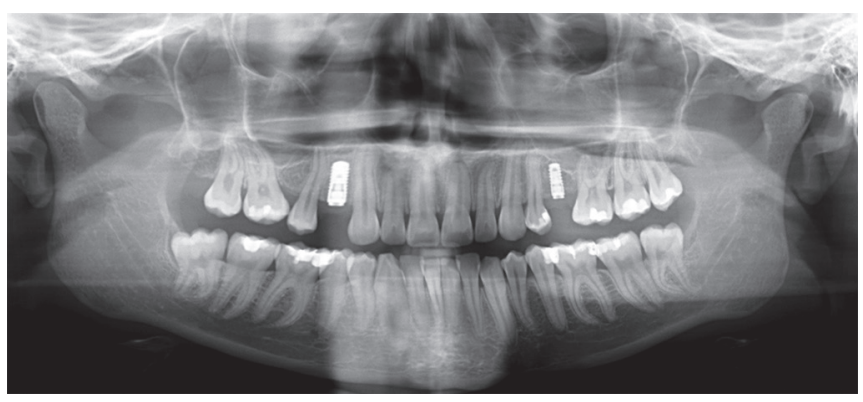

Fig. 3: Panoramic radiograph after surgical procedure in area 14 inserted implant at the location of the first maxillary premolar right with $10 \mathrm{~mm}$ length and $4.1 \mathrm{~mm}$ diameter 
Table 2: Implant length planned on PR or CBCT compared with the conducted surgical procedure

\begin{tabular}{|c|c|c|c|}
\hline & $\begin{array}{l}\text { Equal to } \\
\text { surgery }\end{array}$ & Longer & Shorter \\
\hline \multicolumn{4}{|c|}{ Maxilla $(n=312)$} \\
\hline PR & $28.9 \%(90)^{*}$ & $53.8 \%(168)^{\star *}$ & $17.3 \%(54)$ \\
\hline СBCT & $42.6 \%(133)$ & $41.7 \%(130)$ & $15.7 \%(49)$ \\
\hline \multicolumn{4}{|c|}{${ }^{*} p=0.134 ;{ }^{* *} p=0.134$} \\
\hline \multicolumn{4}{|c|}{ Mandible $(n=360)$} \\
\hline PR & $39.2 \%(141)^{*}$ & $46.1 \%(166)^{\star *}$ & $14.7 \%(53)$ \\
\hline СВCT & $48.9 \%(176)$ & $33.6 \%(121)$ & $17.5 \%(63)$ \\
\hline \multicolumn{4}{|c|}{${ }^{*} p=0.006 ;{ }^{* *} p=0.008 \mathrm{C}$} \\
\hline \multicolumn{4}{|c|}{ Both jaws $(n=672)$} \\
\hline PR & $34.4 \%(231)^{*}$ & $49.7 \%(334)^{\star *}$ & $15.9 \%(107)$ \\
\hline СBCT & $46.0 \%(309)$ & $37.4 \%(251)$ & $16.6 \%(112)$ \\
\hline \multicolumn{4}{|c|}{${ }^{*} p=0.001 ;{ }^{* *} p=0.001$} \\
\hline
\end{tabular}

analyzed in comparison with the conducted surgical procedure and categorized into "equal," "longer/wider," and "shorter/narrower." Ratings with no planning were not considered to calculate the modes.

To explore the magnitude of variability of bone height for various random factors, a linear mixed-effects model was computed. The dependent variable was bone height. The parameter raters, imaging method, jaws, and site were treated as independent random factors. No fixed factor, but an overall mean (intercept) was included for each random factor, the variance with standard deviation (SD) was analyzed. Intra- and interrater agreement was calculated using Cohen's kappa. The level of significance was set at $\alpha=0.05$. All calculations were performed with R 2.15.1. ${ }^{18}$

\section{RESULTS}

\section{Comparison of Suggested Implant Length based on PR or CBCT}

The suggested implant length according to PR or CBCT did not differ in $67.1 \%$ of the sites (Table 1). In $8.8 \%$ of the cases, CBCT-based planning yielded a longer implant than planning based on PR. Vice versa, PR resulted in $24.2 \%$ in longer implants compared with CBCT-based planning. Differences were more pronounced for planning in the mandible $(p=0.001)$ than for the maxilla $(\mathrm{p}=0.134$, Table 1$)$.

\section{Comparison of Suggested Implant Length (based on PR or CBCT) and Conducted Surgical Procedure}

A complete agreement between both radiographic methods and the surgical procedure was achieved in
$28.3 \%$. While an agreement between treatment plan and surgery was found in $34.4 \%$ of the sites planned with PRs, the agreement amounted to $46.0 \%$ when planning was based on CBCT scans (Table 2). The PR planning resulted in an overestimated implant length in $49.7 \%$ of the cases, while in $15.9 \%$ of the sites, implant length was underestimated. For $\mathrm{CBCT}$, an overestimation of the actual implant length was found in $37.4 \%$ of the cases, whereas an underestimation was computed for $16.6 \%$. When the results were separated by jaw, a significant difference between planning and surgical procedure was documented in the mandible, but not in the maxilla (Table 2).

\section{Comparison of Suggested Implant Diameter based on PR or CBCT}

In $44.8 \%$ of the sites, the observers were not able to select an implant diameter based on PR. In 29.7\%, CBCT-based planning yielded a wider implant than planning based on PR. Vice versa, PR resulted in $19.9 \%$ in a wider implant than based on CBCT. The results were more balanced for the mandibular than for the maxilla (Table 1). While there was no significant difference between the two methods in the maxilla, the suggested treatment plan differed significantly in the mandible $(\mathrm{p}<0.05)$.

\section{Comparison of Suggested Implant Diameter (based on PR or CBCT) and Conducted Surgical Procedure}

While an agreement with the surgical procedure was found in $24.4 \%$ of the sites planned with PR, the agreement amounted to $55.7 \%$ when planning was based on CBCT scans (Table 2). Planning on PR underestimated implant diameter in $19.8 \%$ of the cases, while an overestimation occurred in $11.0 \%$ of the sites. For CBCT, an overestimation of actual implant diameter was found in $25 \%$ of the cases, whereas an underestimation was computed in $17.4 \%$.

When the results were separated for the upper and the lower jaw, similar observations were made, except for the planning based on PR for the maxilla. Only $24.4 \%$ of the investigated sites showed a similar implant diameter planned on PR and the implant diameter selected during the surgery. In $19.8 \%$, the implant diameter was planned less invasive on PR than conducted during surgery (Table 3).

\section{Agreement within Observers and between Observers}

The interrater agreement (between rater) expressed as Cohen's kappa was 0.21 for planning implant length on PR and 0.20 for CBCT planning. For implant diameter, the interrater agreement revealed a kappa value of -0.11 
Table 3: Implant diameter planned on PR or CBCT compared with the conducted surgical procedure

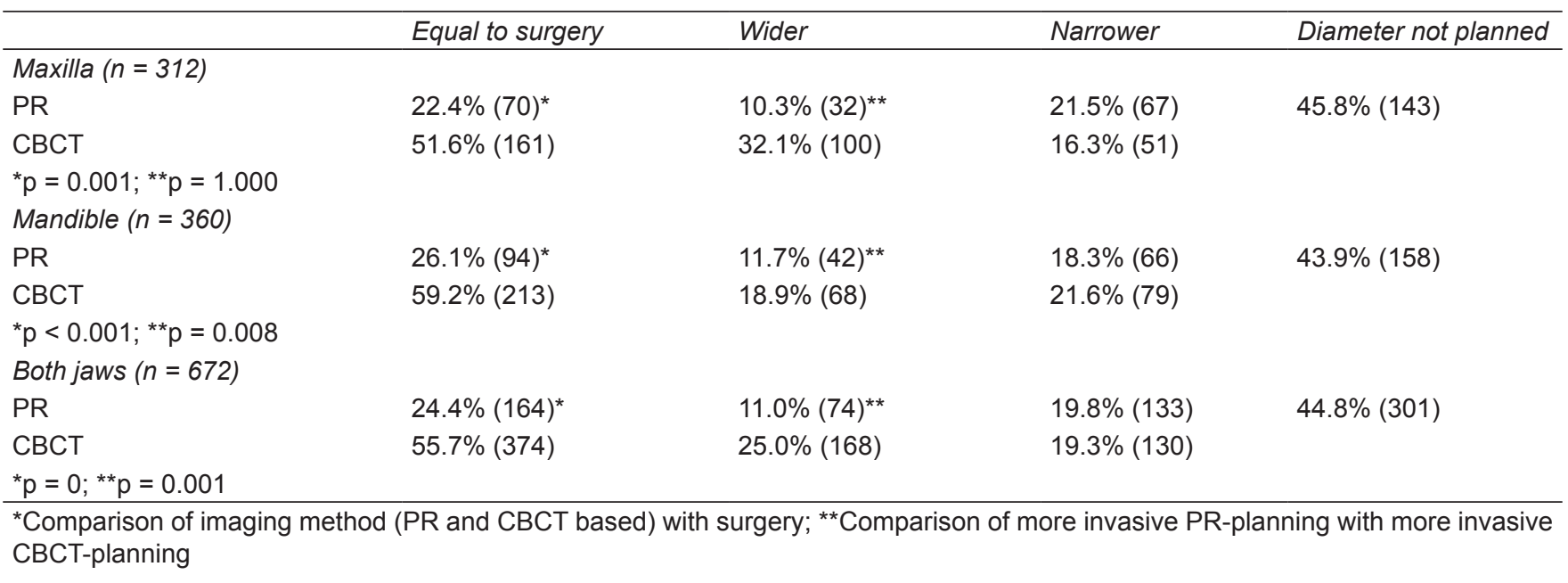

Table 4: Variance analysis of influencing factors affecting bone height

\begin{tabular}{ll}
\hline Variability & Variance $(S D)$ \\
\hline Between modalities & $22.57(4.75)$ \\
Between sites & $18.35(4.28)$ \\
Within rater & $9.10(3.02)$ \\
Between jaws & $9.07(3.01)$ \\
Between rater & $0.13(0.35)$ \\
\hline
\end{tabular}

concerning the planning based on PR vs 0.16 for CBCT images. When comparing the two repetitions each observer had conducted, the planned implant length revealed a within-rater Cohen's kappa of 0.71 for PR vs 0.54 for CBCT. For implant diameter, the kappa values were 0.26 on PR and 0.56 on CBCT images.

\section{Influence on Selection of Implant Dimensions}

According to the linear mixed-effect models, greatest variance in bone height measurements was demonstrated for the type of imaging (PR or CBCT) with variance $( \pm \mathrm{SD})$ of $22.57( \pm 4.7)$. This was followed by the implant sites $(18.35 \pm 4.3)$, while intraobserver agreement, jaw location (maxilla or mandibular), and interrater agreement had minor influence (Table 4).

\section{DISCUSSION}

The aim of this level 3 study was to investigate the impact of modality of radiographic imaging on the implant selection during treatment planning. The analysis revealed that greater implant length was planned based on PR, while wider implants were planned based on CBCTs. A higher agreement occurred between CBCT-based planning and the conducted surgical procedure.

In general, implant selection depends on local bone height and width as well as on the intraoperative surgical situation possibly requiring bone augmentation. In
Table 5: Observers' evaluation of the case shown in Figures 1 and 2

\begin{tabular}{llllll}
\hline & & $\begin{array}{l}\text { Implant } \\
\text { length in }\end{array}$ & $\begin{array}{l}\text { Implant } \\
\text { length } \\
\text { in CBCT }\end{array}$ & $\begin{array}{l}\text { Implant } \\
\text { diameter }\end{array}$ & $\begin{array}{l}\text { Implant } \\
\text { diameter } \\
\text { Observer }\end{array}$ \\
\hline 1 & 1 & 10 & 10 & - & 4.1 \\
2 & 1 & 12 & 10 & 4.1 & 3.3 \\
2 & 1 & 12 & 12 & - & 4.1 \\
3 & 1 & 12 & 12 & - & 3.3 \\
4 & 1 & 12 & 10 & - & 3.3 \\
5 & 1 & 12 & 12 & 4.1 & 3.3 \\
6 & 1 & &
\end{tabular}

Data from first assessment shown. During surgery, an implant with $10 \mathrm{~mm}$ length and $4.1 \mathrm{~mm}$ diameter was inserted

the current study, implant dimensions were selected among predefined lengths and widths, and the term "invasive" was related to greater length or width. Comparing implant planning based on the two different imaging methods, implant length was consistent for approximately two-thirds of the investigated sites, while shorter implants were more frequently planned in CBCT compared with PR, which is in agreement with data from the literature. ${ }^{14,15}$ Based on PR, implant length was overestimated in approximately half of the cases when compared with the conducted surgical procedure, which indicates a certain risk of overseeing morphological variations, such as buccal concavities in the maxilla or lingual undercuts in the posterior mandible. ${ }^{19,20}$ It is well known that panoramic imaging results in distortion and magnification of anatomical structures, and the individual anatomical inclination of the jaw cannot be visualized. Due to these aspects, 2D visualization of bone morphology and anatomical structures in PR is limited, e.g., in the mandible with deficient imaging of the mandibular canal or lingual undercuts (Table 5). Consequently, bone height measurements in PR provide only an estimation of the true height. ${ }^{21-24}$ 
As the number of diameter estimations based on PRs was low, the current results should be considered as an approximation, which need to be verified in further studies. If implant diameter was determined in PR, it was smaller compared with the planning based on CBCT. In contrast to these results, some studies documented either no differences in planning implant diameter, ${ }^{14}$ or planning of narrower implants based on CBCT compared with PR. ${ }^{15}$ This discrepancy reflects different treatment strategies either favoring wider implant diameter with the need for bone in certain cases augmentation, or preferring smaller implants to avoid horizontal augmentation procedures.

Data of the current study revealed that CBCT-based implant planning was more congruent with the surgical protocol than the PR planning. These results are in agreement with previous studies documenting that the content and quality of 3D CBCT images were more precise compared with PRs. ${ }^{9,25}$ However, implant planning based on $\mathrm{CBCT}$ tended to overestimate implant length in more than one-third and implant diameter in $25 \%$. These findings are possibly related to existing differences between the accurate visualization of anatomical structures in CBCT and the real tissues of the intraoperative situs. Even clinical information, which are provided with casts combined with radiographs cannot bridge the discrepancy between images and the actual clinical condition in all cases. ${ }^{17}$

When discussing the adequate indication for imaging techniques, their true benefits and effective dose have to be balanced. As a rough rule-of-thumb, the average effective dose of PR is about 10 times less than the average of effective dose of CBCT scans. ${ }^{26} \mathrm{~A}$ recent meta-analysis ${ }^{27}$ computed mean effective doses between $84 \mu \mathrm{Sv}$ (small field of view) and $212 \mu \mathrm{Sv}$ (small field of view). When comparing these values with an average effective dose from PR ranging between 2.7 and $24.3 \mu \mathrm{Sv}^{28}$ the rule-ofthumb relation still appears valid. New technologies with different scanning protocols and especially comparison of equal volume sizes have to be taken into account for future rule-of-thumb relation. In addition to that, the availability of imaging modalities might also influence the choice of methods, even though with meticulous techniques, panoramic imaging can still be useful. ${ }^{5}$ Furthermore, both modalities possess a wide range of image quality. The selected voxel were in the sub millimeter range with $0.125 \mathrm{~mm}$ for Accuitomo 80 and $0.2 \mathrm{~mm}$ for Kavo 3Dexam. The differences in millimeter between the implants with respect to the possible length and/or diameter are larger than the differences in resolution in terms of voxel. Therefore, an influence of the CBCT apparatus seems to be negligible. Further studies are required to address this point. It has to be kept in mind that the radiographic imaging in living subjects is limited to a realistic visualized detail size of $0.5 \mathrm{~mm} .{ }^{29}$
Recently, treatment planning was analyzed in questionnaire studies with dental practitioners. ${ }^{30,31}$ Both studies showed that there is a great variance regarding the treatment plan selected by a dentist for a given clinical situation. The suggested approach was affected by confounders, such as profession, age or gender. This research indicates that transfer from research to clinical practice may need to be improved and evidence-based guidelines established if possible. ${ }^{32,33}$ However, in evidence-based dentistry settings, the experience of the dental practitioner is an important parameter for decision-making. ${ }^{32}$ In many cases, the variance between different dentists does not necessarily represent the selection of a right vs a wrong approach. It simply reflects clinical reality. Keeping this in mind, the current study was designed and the data regarding variance were provided. Reading panoramic images and planning implants based on PR is a common and routine procedure and dentists are familiar with this method. Reading CBCT scans, even for experienced specialists in implantology, is more challenging. In the current study, the variance of the results concerning CBCT planning was higher compared with the planning based on panoramic images. The interrater agreement (Cohen's kappa: $0.26-0.71)^{33}$ was relatively low, reflecting the great diversity regarding treatment planning of different experienced observers. In a similar study, presurgical implant planning showed an interobserver agreement (kappa) of 0.30 to $0.34 .{ }^{14}$ The observer's effect on interpretation of radiographs is a multifactorial procedure with subjective elements affecting the outcome. ${ }^{34}$ These aspects are still valid and also count for modern 3D imaging technology. In the current study, six experienced observers were selected, who conducted the challenge of data interpretation in terms of diagnostic efficacy. All images were provided with full-options in changing the settings, which means that each observer could rotate the CBCT volume in her/his appropriate way for implant treatment planning. Six observers represent the process of implant planning more appropriate than just one observer, who might have a personal opinion of implant planning. Implant length and diameter were planned in real cases, with the same records the surgeon had for the entire implantation process.

Since this was a study with the primary focus on treatment planning, in this study, the observers were not able to operate the cases themselves. However, an alternative study design would follow surgeons during the planning phase based on both radiographic methods and observe their actual surgical procedures. However, such a protocol would only consider the planning process of single subjects and thus would preclude a multirater analysis, which is a strength of our study. 


\section{CONCLUSION}

The results of the current study indicate that bone height measurements in presurgical implant planning are influenced by the imaging modality (PR and CBCT scan). Compared with PR planning, CBCT-based implant planning was closer related to the conducted surgery; however, interindividual differences were also higher.

\section{CLINICAL SIGNIFICANCE}

The CBCT scans provided sufficient additional information in the third dimension to facilitate reliable planning of the implant dimension, particularly the implant diameter.

\section{ACKNOWLEDGMENTS}

The authors gratefully acknowledge the time of the observers Dan Brüllmann, Kristian Knierim, Sebastian Kühl, Julia Mennickheim, Anastasia Mouratidou, and Stefan Wentaschek. They also gratefully acknowledge the support by Andreas Schötzau and Christian Müller with the statistical analysis. Author Dagassan-Berndt would like to thank "Freiwillige Akademische Gesellschaft Basel" for the financial support during the project

\section{REFERENCES}

1. Shabazian M, Vandewoude C, Wyatt J, Jacobs R. Comparative assessment of panoramic radiography and $\mathrm{CBCT}$ imaging for radiodiagnostics in the posterior maxilla. Clin Oral Investig 2014 Jan;18(1):293-300.

2. Tadinada A, Fung K, Thacker S, Mahdian M, Jadhav A, Schincaglia GP. Radiographic evaluation of the maxillary sinus prior to dental implant therapy: a comparison between two-dimensional and three-dimensional radiographic imaging. Imaging Sci Dent 2015 Sep;45(3):169-174.

3. Tyndall DA, Price JB, Tetradis S, Ganz SD, Hildebolt C, Scarfe WC; American Academy of Oral and Maxillofacial Radiology. Position statement of the American Academy of Oral and Maxillofacial Radiology on selection criteria for the use of radiology in dental implantology with emphasis on cone beam computed tomography. Oral Surg Oral Med Oral Pathol Oral Radiol 2012;113(6):817-826.

4. Academy of Osseointegration. 2010 Guidelines of the Academy of Osseointegration for the provision of dental implants and associated patient care. Int J Oral Maxillofac Implants 2010 May-Jun;25(3):620-627.

5. Harris D, Horner K, Gröndahl K, Jacobs R, Helmrot E, Benic GI, Bornstein MM, Dawood A, Quirynen M. E.A.O. guidelines for the use of diagnostic imaging in implant dentistry 2011. A consensus workshop organized by the European Association for Osseointegration at the Medical University of Warsaw. Clin Oral Implants Res 2012 Nov;23(11):1243-1253.

6. Dula K, Bornstein MM, Buser D, Dagassan-Berndt D, Ettlin DA, Filippi A, Gabioud F, Katsaros C, Krastl G, Lambrecht JT, et al. SADMFR guidelines for the use of Cone-Beam Computed Tomography/Digital Volume Tomography. Swiss Dent J 2014;124(11):1169-1183.
7. Bornstein MM, Horner K, Jacobs R. Use of cone beam computed tomography in implant dentistry: current concepts, indications and limitations for clinical practice and research. Periodontol 20002017 Feb;73(1):51-72.

8. Vazquez L, Saulacic N, Belser U, Bernard JP. Efficacy of panoramic radiographs in the preoperative planning of posterior mandibular implants: a prospective clinical study of 1527 consecutively treated patients. Clin Oral Implants Res 2008 Jan;19(1):81-85.

9. Angelopoulos C, Thomas S, Hechler S, Parissis N, Hlavacek M. Comparison between digital panoramic radiography and cone-beam computed tomography for the identification of the mandibular canal as part of presurgical dental implant assessment. J Oral Maxillofac Surg 2008 Oct;66(10):2130-2135.

10. Gerlach NL, Meijer GJ, Maal TJ, Mulder J, Rangel FA, Borstlap WA, Berge SJ. Reproducibility of 3 different tracing methods based on cone beam computed tomography in determining the anatomical position of the mandibular canal. J Oral Maxillofac Surg 2010 Apr;68(4):811-817.

11. SEDENTEXCT. Radiation protection: Cone beam CT for dental and maxillofacial radiology. Evidence based guidelines. Manchester: SEDENTEXCT; 2011. pp. 1-139. [cited 2012 Mar]. Available from: http: / / www.sedentexct.eu/files / guidelines_final.pdf.

12. Fryback DG, Thornbury JR. The efficacy of diagnostic imaging. Med Decis Making 1991 Apr-Jun;11(2):88-94.

13. Schulze R. Editorial: dentomaxillofacial radiology. Dentomaxillofac Radiol 2012 Jan;41(1):1-2.

14. Guerrero ME, Noriega J, Castro C, Jacobs R. Does cone-beam $\mathrm{CT}$ alter treatment plans? Comparison of preoperative implant planning using panoramic versus cone-beam CT images. Imaging Sci Dent 2014 Jun;44(2):121-128.

15. Correa RL, Spin-Neto R, Stavropoulos A, Schropp L, da Silveira HE, Wenzel A. Planning of dental implant size with digital panoramic radiographs, CBCT-generated panoramic images and CBCT cross-sectional images. Clin Oral Implants Res 2014 Jun;25(6):690-695.

16. Dagassan-Berndt DC, Zitzmann NU, Walter C, Schulze RK. Implant treatment planning regarding augmentation procedures: panoramic radiographs vs cone beam computed tomography images. Clin Oral Implants Res 2016 Aug;27(8):1010-1016.

17. Jensen C, Raghoebar GM, Meijer HJ, Schepers R, Cune MS. Comparing two diagnostic procedures in planning dental implants to support a mandibular free-ending removable partial denture. Clin Implant Dent Relat Res 2016 Aug;18(4):678-685.

18. R Core Team. R: a language and environment for statistical computing. Vienna: R Foundation for Statistical Computing; 2012. Available from: http://www.R-project.org/.

19. Vazquez L, Nizam Al Din Y, Christoph Belser U, Combescure C, Bernard JP. Reliability of the vertical magnification factor on panoramic radiographs: clinical implications for posterior mandibular implants. Clin Oral Implants Res 2011 Dec;22(12):1420-1425.

20. Nickenig JH, Wichmann M, Eitner S, Zöller JE, Kreppel M. Lingual concavities in the mandible: a morphological study using cross-sectional analysis determined by CBCT. J Craniomaxillofac Surg 2015 Mar;43(2):254-259.

21. Tronje G, Eliasson S, Julin P, Welander U. Image distortion in rotational panoramic radiography. II. Vertical distances. Acta Radiol Diagn (Stockh) 1981 Jul;22(4):449-455. 
22. Tronje G, Welander U, McDavid WD, Morris CR. Imaging characteristics of seven panoramic X-ray units, Part IV. Horizontal and vertical magnification. Dentomaxillofac Radiol 1985;(Suppl 8):29-34.

23. Welander U, McDavid WD, Tronje G, Morris CR. Imaging characteristics of seven panoramic x-ray units. Part V. Image distortion in planes at different object depths. Dentomaxillofac Radiol 1985;(Suppl 8):35-43.

24. Devlin H, Yuan J. Object position and image magnification in dental panoramic radiography: a theoretical analysis. Dentomaxillofac Radiol 2013 Aug;42(1):29951683.

25. Loubele M, Van Assche N, Carpentier K, Maes F, Jacobs R, van Steenberghe D, Suetens P. Comparative localized linear accuracy of small-field cone-beam CT and multislice CT for alveolar bone measurements. Oral Surg Oral Med Oral Pathol Oral Radiol Endod 2008 Apr;105(4):512-518.

26. Schulze R. Strahlendosis bei der röntgenologischen Bildgebung für implantologische Fragestellungen im Vergleich: Intraoral-, Panoramaschichtaufnahme, DVT und CT. Implantologie 2009 Dec;17(4):377-386.

27. Ludlow JB, Timothy R, Walker C, Hunter R, Benavides E, Samuelson DB, Scheske MJ. Effective dose of dental CBCT-a meta analysis of published data and additional data for nine CBCT units. Dentomaxillofac Radiol 2015 Jan;44(1):20140197.
28. European Commission. Radiation Protection no 172: cone beam CT for dental and maxillofacial radiology. Evidence based guidelines. Manchester: Sedentexct Project; 2012

29. Brüllmann D, Schulze RK. Spatial resolution in CBCT machines for dental/maxillofacial applications-what do we know today? Dentomaxillofac Radiol 2015 Jan;44(1): 20140204.

30. Junges R, Zitzmann NU, Walter C, Rösing CK. Dental care providers' decision making regarding maintenance of compromised teeth and implant therapy indication: an analysis of gender and enrollment in teaching positions. Clin Oral Implants Res 2014 Sep;25(9):1027-1033.

31. Zitzmann NU, Zemp E, Weiger R, Lang NP, Walter C. Does a clinician's sex influence treatment decisions? Int J Prosthodont 2011 Nov-Dec;24(6):507-514.

32. Bonetti D. Guideline improved dentists' knowledge but not their clinical decision-making skills. Evid Based Dent 2006 Mar;7:8.

33. Fleiss, JL. Statistical methods for rates and proportions. 2nd ed. New York: John Wiley; 1981.

34. Goldman M, Pearson AH, Darzenta N. Endodontic successwho's reading the radiograph? Oral Surg Oral Pathol Oral Med 1972 Mar;33(3):432-437. 